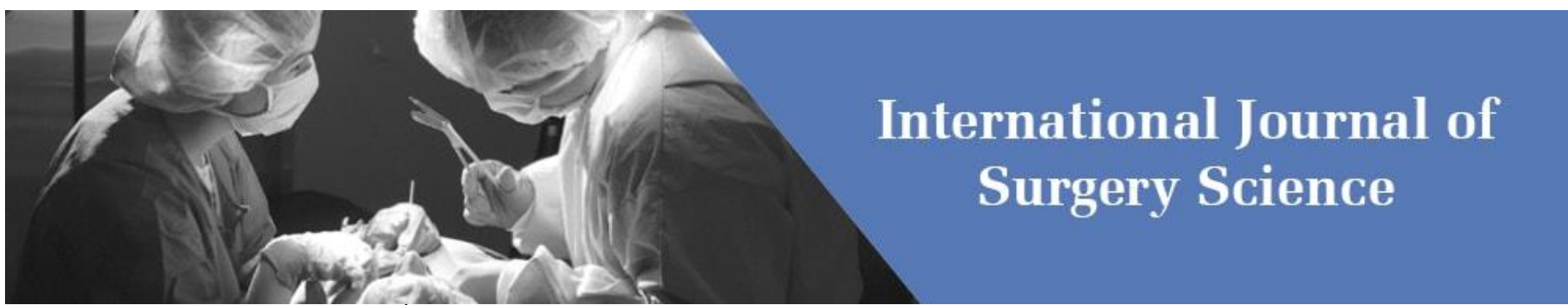

E-ISSN: 2616-3470

P-ISSN: 2616-3462

(C) Surgery Science

www.surgeryscience.com

2019 ; 3(4): 329-331

Received: 11-08-2019

Accepted: 15-09-2019

Shyam Sundar Tandri

Assistant Professor, Department of

General Surgery, Narayana

Medical College, Nellore, Andhra

Pradesh, India

Arjun

Post Graduate, Department of

General Surgery, Narayana

Medical College, Nellore, Andhra

Pradesh, India

Mahidhar Reddy Venkatapuram Professor, Department of General Surgery, Narayana Medical College, Nellore, Andhra Pradesh, India

Corresponding Author:

Mahidhar Reddy Venkatapuram

Professor, Department of General

Surgery, Narayana Medical

College, Nellore, Andhra Pradesh,

India

\section{Effectiveness of open pre-peritoneal approach in the treatment of inguinal hernia in adults}

\author{
Shyam Sundar Tandri, Arjun and Mahidhar Reddy Venkatapuram
}

DOI: https://doi.org/10.33545/surgery.2019.v3.i4f.263

\section{Abstract}

Background: Bassini (1844-1924) transformed inguinal hernia repair into a successful venture with minimal morbidity. The success of the Bassini repair over its predecessors ushered in an era of tissue-based repairs. Inguinal hernia repair underwent its most recent transformation. Laparoscopic inguinal hernia repair as the Trans Abdominal Pre Peritoneal (TAPP) repair, and the Totally Extra Peritoneal repair (TEP). The most significant advances to inguinal hernia have been repair through open pre-peritoneal approach. The advantage of pre-peritoneal approach is that the prosthetics can be placed between hernial contents and hernial defects. The need for present study is to review the effectiveness and complications of open preperitoneal approach in inguinal hernia repair.

Methods: A prospective observational study in patients who had undergone open pre-peritoneal mesh repair in Narayana medical college, Nellore. Sample size- a total of 50 patients are taken in this study.

Results: In this present study 50 cases of inguinal hernia were taken and operated by open pre-peritoneal approach.

Conclusion: In this present study the effectiveness of pre-peritoneal approach in Inguinal hernia was studied in terms of post-operative pain, wound complications, time to return to pre-operative physical activities and short term recurrence. There was no recurrence rate on follow-up, Post operative pain was low compared to other approaches from various studies, and time to return to normal activities was significantly less than the other repairs.

Keywords: Effectiveness, pre-peritoneal, inguinal hernia

\section{Introduction}

Bassini (1844-1924) transformed inguinal hernia repair into a successful venture with minimal morbidity. The success of the Bassini repair over its predecessors ushered in an era of tissuebased repairs. Modifications of the Bassini repair were manifest in the Mc-Vay and Shouldice repairs. All three of these techniques, as well as modern variations such as the Desarda operation, are currently practiced ${ }^{[1]}$.

In the early 1980s, Lichtenstein popularized the tension free repair, supplanting tissue-based repairs with the widespread acceptance of prosthetic materials for posterior wall of inguinal canal reconstruction. This technique was superior to previous tissue-based repair in that mesh could restore the strength of the transversalis fascia, thereby avoiding tension in the defect closure.

Inguinal hernia repair underwent its most recent transformation. Laparoscopic inguinal hernia repair as the Trans Abdominal Pre Peritoneal (TAPP) repair, and the Totally Extra Peritoneal repair (TEP).

Despite the frequency of this procedure, no surgeon has ideal results, and complications such as postoperative pain, nerve injury, infection, and recurrence remain ${ }^{[2]}$. In spite of these, inguinal hernia still remains unconquered and poses a lot of challenges for all surgeons practicing hernia repair.

There are more than 200 techniques for inguinal hernia repair, and every technique author claiming that their technique is superior to others.

In the early 1980s, Lichtenstein popularized the tension free repair, supplanting tissue-based repairs with the widespread acceptance of prosthetic materials for posterior wall of inguinal canal reconstruction. This technique was superior to previous tissue-based repair in that mesh could restore the strength of the transversalis fascia, thereby avoiding tension in the defect closure. 
Inguinal hernia repair underwent its most recent transformation. Laparoscopic inguinal hernia repair as the Trans Abdominal Pre Peritoneal (TAPP) repair, and the Totally Extra Peritoneal repair (TEP) ${ }^{[3]}$.

Despite the frequency of this procedure, no surgeon has ideal results, and complications such as postoperative pain, nerve injury, infection, and recurrence remain6. In spite of these, inguinal hernia still remains unconquered and poses a lot of challenges for all surgeons practicing hernia repair.

There are more than 200 techniques for inguinal hernia repair, and every technique author claiming that their technique is superior to others.

\section{Origin of pre-peritoneal approach}

The most significant advances to inguinal hernia have been repair through open pre-peritoneal approach.

The advantage of pre-peritoneal approach is that the prosthetics can be placed between hernial contents and hernial defects

The posterior approach to the repair of inguinal and femoral hernia had its origin with annandale in 18764 . Since then interest in its use has waxed and waned, primarly because of a lack of understanding of the groin anatomy as seen posteriorly.

This usually practiced anterior approach has only access to superior portion of Myopectineal orifice of Fruchaud where as complete access is only possible only through posterior $\operatorname{approach}^{[5]}$.

The need for present study is to review the effectiveness and complications of open pre-peritoneal approach in inguinal hernia repair.

\section{Aims and Objectives}

Aims: to study the effectiveness of the preperitoneal open mesh inguinal hernia repair.

Objectives: To study the short term efficacy of pre-peritoneal open mesh repair of inguinal hernia in terms of:

- Post operative pain.

- Post operative complications like seroma and hematoma formation and wound infection.

- Time to return to pre-operative activities after surgery.

- Short term Recurrences.

\section{Materials and Methods}

- Source of data- Patients admitted with Inguinal Hernia in surgical ward (direct/Indirect, Unilateral or Bilateral) at Narayana Medical College and Hospital Nellore, between January 2018-2019.

- Research design-A prospective observational study in patients who had undergone open pre-peritoneal mesh repair in Narayana medical college, Nellore.

- Sample size- a total of 50 patients are taken in this study.

- An informed consent was taken from all patients.

- Open-Preperitoneal mesh repair was performed by the same surgical team.

- Institutional ethical committee approval was taken prior to commencement of study.

Since most of patients in this study were not having specified work or students, the time taken to attain the pre-operative physical activity level was recorded in days beginning from the day of surgery. And time to return to pre operative physical status data was obtained after 1 week on first follow-up.
Postoperative complications like seroma formation and hematoma formation were recorded and tabulated.

Visual analogue scale was used to assess the post operative pain score.

All patients were followed up for minimum of 6 months and maximum of 2 years for early recurrence.

\section{Inclusion criteria}

- Subjects aged between 18 to 70 .

- Should have a inguinal hernia.

- Patients Fit for anaesthesia

- Consent to participate in study

\section{Exclusion criteria}

- Other hernias of anterior abdominal wall.

- Unfit for anesthesia

- Complicated hernias like-strangulated, Incarcerated inguinal hernia

- Previously operated through open pre-Peritoneal mesh repair.

- Patients below 18 years and above 70 years were excluded from the study.

\section{Results}

In this present study 50 cases of inguinal hernia were taken and operated by open pre-peritoneal approach and the following observations and results were noted.

Table 1: Incidence of Type of Hernia (N=50)

\begin{tabular}{|c|c|c|}
\hline Type of hernia & No of cases & Percentage \\
\hline Indirect inguinal hernia & 30 & $60 \%$ \\
\hline Direct inguinal hernia & 12 & $24 \%$ \\
\hline Bilateral indirect inguinal hernia & 8 & $16 \%$ \\
\hline Total & 50 & $100 \%$ \\
\hline
\end{tabular}

Table 2: Comparision of age distribution $(\mathrm{N}=50)$

\begin{tabular}{|c|c|c|}
\hline Age & No of cases & Percentage \\
\hline $18-25$ & 8 & $16 \%$ \\
\hline $26-35$ & 13 & $26 \%$ \\
\hline $36-45$ & 9 & $18 \%$ \\
\hline $46-55$ & 8 & $16 \%$ \\
\hline $56-65$ & 8 & $16 \%$ \\
\hline $66-70$ & 4 & $8 \%$ \\
\hline Total & 50 & $100 \%$ \\
\hline
\end{tabular}

Table 3: Sex Distribution (N=50)

\begin{tabular}{|c|c|c|}
\hline Sex & No of cases & Percentage \\
\hline Male & 49 & $98 \%$ \\
\hline Female & 1 & $2 \%$ \\
\hline Total & 50 & $100 \%$ \\
\hline
\end{tabular}

Table 4: Comparison of Post Operative Pain Assessment - Vas Scale $(\mathrm{N}=50)$

\begin{tabular}{|c|c|c|}
\hline Visual analogue scale & $\begin{array}{c}\text { No of patients on } \\
\mathbf{1}^{\text {st }} \text { POD }\end{array}$ & $\begin{array}{c}\text { No of patients on 5 } \\
\text { POD }\end{array}$ \\
\hline$<3$ & 8 & 35 \\
\hline $3-5$ & 15 & 8 \\
\hline $5-8$ & 14 & 6 \\
\hline$>8$ & 13 & 1 \\
\hline Total & 50 & 50 \\
\hline
\end{tabular}


Table 5: Time to Return to Pre-Operative Physical Activity ( $\mathrm{N}=50$ )

\begin{tabular}{|c|c|}
\hline Number of days & Number of patients \\
\hline 2 & $24(48 \%)$ \\
\hline 3 & $14(28 \%)$ \\
\hline 4 & $8(16 \%)$ \\
\hline 5 & $4(8 \%)$ \\
\hline Average-2.84 Days & Total Patients-50 \\
\hline
\end{tabular}

Table 6: Incidence of Complications-Seroma and Hematoma

\begin{tabular}{|c|c|c|}
\hline Post Operative Complications & No of patients & Percentage \\
\hline Seroma formation & 9 & $18 \%$ \\
\hline Hematoma formation & 3 & $14 \%$ \\
\hline
\end{tabular}

\section{Discussion}

In this present study total 50 patients of inguinal hernia are operated through open pre-peritoneal approach in Narayana medical college, Nellore.

This study demonstrates the effectiveness of open pre-peritoneal approach in treatment of inguinal hernia in adults. Inguinal hernia is a common problem in males, and surgery is the only treatment of choice. So the quest to find an efficient and easy to perform technique which has low rates of complications and no recurrence has been a surgeons dream.

In present study there was nil recurrence rates and post operative recovery is short compared to other approaches, the mean time taken to return to pre-operative physical status after discharge was 2.84 days, $9(18 \%)$ patients had seroma formation which was managed conservatively, and $3(6 \%)$ patients had hematoma formation. None of the patients had wound infection.

The surgical technique found to be comparable to the other modern techniques of inguinal hernia repairs like Lichtenstein's technique ${ }^{[6]}$ or laparoscopic technique ${ }^{[7]}$ in terms of post operative pain, time taken to regain pre-operative physical activity level and short term recurrence.

In this present study the effectivenss of pre-peritoneal approach in inguinal hernia was studied in terms of post-operative pain, wound complications, time to return to pre-operative physical activities and short term recurrence. 50 patients with inguinal hernia were studied in this group and operated by the same surgical team evaluated after.

There was no recurrence rate on follow-up, Since the follow up period is short this finding requires to be substantiated by long term follow-up.

Post-operative pain was low compared to other approaches from various compared to Lichtenstein approach from various studies and there was no wound infection in this study. Several Studies concluded that open pre-peritoneal approach is the ideal in treatment of recurrent inguinal hernias, which were primarly operated through anterior approach ${ }^{[8]}$.

Hence open pre-peritoneal approach to repair inguinal hernia has becoming a gold standard for surgery, as it has similar advantages as laparoscopic repair in terms of less pain, wound infections, and complete repair of the myo-pectineal orifice of frauchad and lower cost than the laparoscopic repair.

\section{Conclusion}

Open pre-peritoneal approach should be the ideal surgery in treatment of inguinal both primary and recurrent inguinal hernias.

\section{References}

1. Justinp Wagner f. Charles Brunicardi, Parviz K. Schwartzes principles of surgery 10th edition Page No, 1495.

2. Johnson J, Roth JS, Hazey JW et al. The history of open inguinal hernia repair. Curr Surg. 2004; 61:49.

3. Rutkow IM. Demographic and socioeconomic aspects of hernia repair in the United States in 2003. Surg Clin North Am. 2003; 83:1045.

4. Mark Malangoni A, Micheal Rosen J. Chapter 46 Sabiston text book of surgery: the biological basis of modern surgical practice 19th Edition Page, 1114.

5. Gould J. Laparoscopic versus open inguinal hernia repair. Surgical Clinics North America. 2008; 88:1073-1081.

6. Rutkow IM. Epidemiologic, economic, and sociologic aspects of hernia surgery in the United States in the 1990s. Surgical Clinics North America. 1998; 78:941.

7. Desarda MP. Physiological repair of inguinal hernia: a new technique (study of 860 patients). Hernia. 2006; 10:143146.

8. Fischers Master of Surgery Sixth Edition, by m Nyhus-pre peritoneal hernia repair by Nyhus E Chapter, 118. 